\title{
Caracterización fisicoquímica del aceite crudo y refinado de la semilla de Proboscidea parviflora (Uña de gato)
}

\author{
Por M. Ortega Nieblas * y L. Vázquez Moreno \\ Centro de Investigaciones Científicas y Tecnológicas de la Universidad de Sonora. Apartado Postal 1819. \\ Hermosillo, Sonora, México.
}

\section{RESUMEN}

Caracterización fisicoquímica del aceite crudo y refinado de la semilla de Proboscidea parviflora (Uña de gato).

En la búsqueda de semillas que sirvan como nuevas fuentes de oleaginosas para cubrir las necesidades existentes a nivel nacional, se estudió el aceite de Proboscidea parviflora. El aceite se extrajo de la semilla con hexano en un aparato Soxhlet. Se le determinaron sus propiedades físicas y químicas, así como el perfil de ácidos grasos por cromatografía de gases, tanto del aceite crudo como del refinado. Después de un proceso sencillo de refinación y desodorización, el aceite resultó ser de buena calidad con un grado de insaturación relativamente alto.

PALABRAS-CLAVE: Aceite de uña de gato - Proboscidea parviflora Propiedades físico-químicas.

\section{SUMMARY}

Physicochemical characterization of crude and refined oil of Proboscidea parviflora seeds (Uña de gato).

Searching for seeds as new sources of oleaginous that can cover the national needs, it was studied the oil of Proboscidea parviflora. This oil was extracted from the seed with hexane in a Soxhlet apparatus, and its physical and chemical properties were determined, as well as the fatty acid composition by gas chromatography, in crude and refined oil. After a simple refining and deodorization process, the extracted oil had good quality with a high percentage of insatured fatty acids.

KEY-WORDS: Physico-chemical properties- Proboscidea parviflora"Uña de gato" oil.

\section{INTRODUCCION}

Los lípidos son importantes en la nutrición por su alta aportación energética, además de su innegable papel como fuente de ácidos grasos esenciales. Las grasas y aceites comestibles comprenden el 30-40 \% del aporte energético de una dieta equilibrada para el ser humano, sin embargo pueden ser menores del $5 \%$ de la dieta en algunos paises subdesarrollados (6). En los años de 19661980 en los Estados Unidos fue consumido un promedio de 530 calorías de aceite vegetal por persona diariamente, mientras que en Centroamérica y al Norte y Oeste de Africa se consumieron 185 calorías de aceite vegetal por día (4).

En ocasiones esta deficiencia dietética es causada por un suministro inadecuado de aceites vegetales, el proble- ma puede llegar a ser particularmente agudo en países áridos debido a que de los 36 mejores cultivos oleaginosos solo siete (maíz, algodón, olivo, ajonjolí, girasol, cártamo y soya) pueden ser cultivados bajo condiciones relativamente secas (12).

En la actualidad existe la necesidad de buscar nuevas fuentes de oleaginosas debido a que el total de la producción nacional es insuficiente. Una alternativa para resolver este problema es el aprovechamiento de los recursos naturales del desierto de Sonora, en el cual se desarrolla la Proboscidea parviflora, planta comúnmente conocida como "uña de gato"; su semilla fue utilizada por algunas tribus como alimento y parte del fruto como ornamento de cestería (5). Es una planta herbácea anual, cuya semilla entera contiene del 36 al $40 \%$ de aceite y del 23 al $27 \%$ de proteína, por lo que es clasificada como oleaginosa (4); en estudios realizados con la especie Proboscidea parviflora Var. Hohokamiana se reportó que el $36 \%$ del peso de la semilla es aceite comestible con características parecidas al aceite de girasol (7).

\subsection{Proboscidea Parviflora como fuente de aceite}

En experimentos de cultivo en zonas áridas se ha obtenido una producción de $2587 \mathrm{~kg}$ por hectárea, de la cual se puede obtener alrededor de $100 \mathrm{~kg}$ de aceite y $675 \mathrm{~kg}$ de proteína, cantidades que se comparan favorablemente con otros cultivos ya tradicionales (9). Dada la importancia que esta especie tendría al ser domesticada en el Estado de Sonora se consideró de interés determinar las propiedades físicas y químicas del aceite crudo y refinado de Proboscidea parviflora.

\subsection{Descripción botánica}

Uña de gato Proboscidea parviflora (Woot y Stand). Esta planta era conocida por los indígenas en las zonas áridas varias décadas antes de que los científicos se interesaran por su posible cultivo (11). La planta pertenece al orden scropulariales, familia martiniaceae, subgénero Proboscidea que incluye diez especies, entre ellas la Proboscidea parviflora. Es herbácea anual, su raíz raramente excede los $20 \mathrm{~cm}$, los tallos son largos y robustos, 
ramificados, como se muestra en la Fig. 1; el color de la flor depende de la especie (13). El fruto posee un cuerpo que mide casi $2 \mathrm{~cm}$ de diámetro y de 5 a $7 \mathrm{~cm}$ de longitud, tiene un par de cuernos que doblan la longitud del cuerpo, cuenta con unas crestas con dientes lisos e irregulares de 2 a 3 $\mathrm{mm}$ de ancho y de 5 a $7 \mathrm{~mm}$ de longitud, en el centro del cuerpo se encuentra una especie de cámara que contiene las semillas (7). Las semillas son de forma ovoide, un poco angulada, de color negro opaco, tuberculado y verrugoso, el tamaño puede variar dependiendo del acomodo del fruto, llegando a medir de 7 a $11 \mathrm{~mm}$ de diámetro (13).

La uña de gato se desarrolla satisfactoriamente en el desierto de Sonora y Sur de Arizona donde la temperatura promedio en el verano es de $25-37^{\circ} \mathrm{C}$ y altitudes bajas de $150 \mathrm{~m}$ sobre el nivel del mar. Su distribución en el Estado de Sonora es en Sonoyta, Puerto Libertad, Altar, Colonia Morelos, Huachineras, Mozocahui, San Bernardo, Alamos y Ures (10).

\section{PARTE EXPERIMENTAL}

La recolección del fruto se realizó en un terreno localizado dentro del Municipio de Ures, Sonora. Dicho munici-

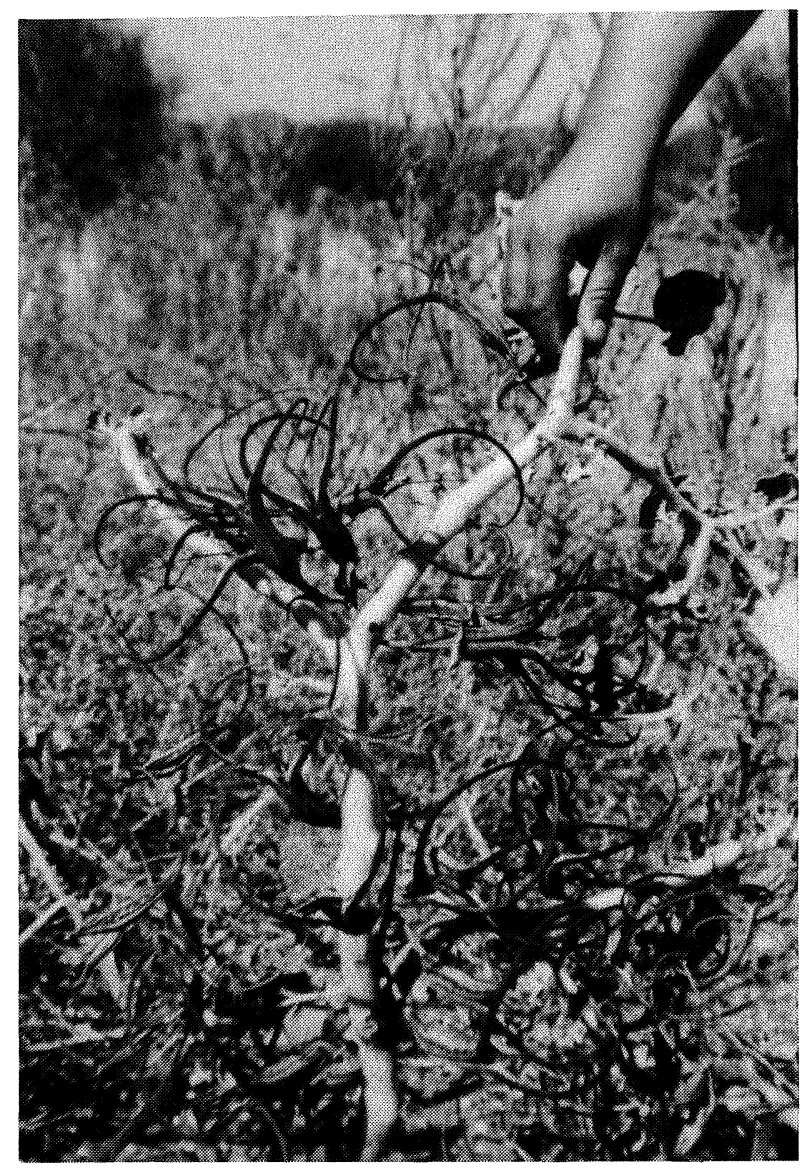

Figura 1

Fruto de Uña de gato (Proboscidea parviflora) en etapa de madurez fisiológica pio se encuentra ubicado geográficamente en los $29^{\circ} 26^{\prime}$ Latitud Norte y 110 Longitud Oeste y a los $342 \mathrm{~m}$ sobre el nivel del mar, donde se recolectaron manualmente los frutos maduros, separando posteriormente las semillas en cantidades necesarias para la obtención del aceite. Todos los análisis se realizaron por triplicado.

\subsection{Extracción y desolventización del aceite}

La semilla fue desquebrajada en una licuadora, secada a $80^{\circ} \mathrm{C}$ por hora, posteriormente molida en un molino Wiley Malla No. 20 para luego ser sometida a una extracción intermitente en un soxhlet con capacidad de sifón de $2200 \mathrm{ml}$ usando hexano como disolvente. La miscela obtenida fue filtrada a través de papel whatman \#1. El hexano fue separado del aceite a presión reducida y a $50^{\circ} \mathrm{C}$, obteniéndose de esta manera el aceite crudo.

\subsection{Refinación del aceite}

El aceite fue neutralizado, adicionando la cantidad requerida de $\mathrm{NaOH} 3 \mathrm{~N}$, a temperatura ambiente y con agitación. Posteriormente se calentó el aceite a $65^{\circ} \mathrm{C}$ a presión reducida durante $15 \mathrm{~min}$, se dejó enfriar en reposo a temperatura ambiente durante una hora, para favorecer la decantación, y se filtró. El aceite neutralizado se lavó con agua desionizada (al $20 \%$ en peso del aceite), se secó a $90^{\circ} \mathrm{C}$ durante 30 min a presión reducida y se enfrió a temperatura ambiente para posteriormente ser analizado.

\subsection{Desodorización}

En este proceso al aceite se le aplicó calentamiento, agitación y vacío. Al alcanzar la temperatura de $200^{\circ} \mathrm{C}$ se le agregó agua en pequeñas proporciones, cuando la temperatura bajó a $180^{\circ} \mathrm{C}$, se adicionó ácido cítrico en igual forma que el agua y finalmente se llevó a temperatura ambiente.

\subsection{Análisis físicos}

Fueron llevados a cabo de acuerdo con los métodos oficiales recomendados por A.O.C.S.

Indice de refracción: método Cc 7-25, utilizando un refractómetro Abbe Bausch \& Lamb modelo 31.

Densidad: método Cc $10 \alpha-25$.

Viscosidad: método Tq 1-64, usando un viscosímetro Saybolt.

Punto de fusión: método Cc 1-25.

Color: método Cc 13-45, usando un colorímetro Lovibond.

\subsection{Análisis químicos}

Los análisis se realizaron de acuerdo a los métodos recomendados por la A.O.C.S., excepto la determinación de 
índice de iodo que se llevó a cabo por el método oficial recomendado por la A.O.A.C.

Indice de lodo: método de Hanus. A.O.A.C. 28. 018. Indice de saponificación: método Cd 8-53.

Acidos grasos libres: método Ca 5-40.

Valor del ácido o índice de acidez: método Cd 3-63.

Carotenoides: método Cd 7-58 A.O.C.S. Se utilizó un espectrofotómetro Beckman modelo 25 y la ecuación de Zcheile.

\subsection{Análisis cromatográficos}

La cuantificación de los ácidos grasos se efectuó por cromatografía de gases (cromatógrafo Varian 6000) utilizando el detector de ionización de llama, con una sensibilidad de $2 \times 10$ AFS, una columna de acero inoxidable de $6 \mathrm{ff} \times 1 / 8$ " con empaquetamiento GP $3 \%$ SP-2310/2\% SP2300 sobre un soporte de $100 / 120$ chromosorb AW, a una temperatura inicial de $190^{\circ} \mathrm{C}$ por 2 minutos hasta una final de $220^{\circ} \mathrm{C}$ con una velocidad de incremento de $2^{\circ} \mathrm{C}$ por minuto y nitrógeno como gas portador a una velocidad de flujo de $20 \mathrm{ml} / \mathrm{min}$. La temperatura del detector fue de $250^{\circ} \mathrm{C}$. Los datos fueron procesados mediante un integrador (Varian CDS 401) adaptado al cromatógrafo. Para la cuantificación de los ácidos grasos fue necesario llevar a cabo como paso previo, una saponificación completa de los triglicéridos con hidróxido de sodio y una metilación de los ácidos grasos (AOCS, Ce 27, 1970).

\section{RESULTADOS Y DISCUSION}

En el proceso de extracción del aceite se obtuvo una eficiencia del $84.8 \%$ siendo éste un rendimiento aceptable. En la Tabla I, se observan las propiedades físicas y químicas del aceite crudo y refinado donde se puede apreciar que al refinar el aceite se obtuvo un aumento en cuanto al contenido en los Indices de lodo y de saponificación, así como una disminución de ácidos grasos libres y peróxidos por lo que se consideró que el proceso de refinación fue satisfactorio. El contenido del Indice de lodo, (115.5), es comparable con el de la mayoría de los aceites vegetales, esto refleja un alto grado de insaturación. Se clasificó como aceite semisecante y se encuentra entre el de ajonjolí y el de aceite de maíz (15).

Se obtuvo un índice de acidez bajo, esto junto con la baja pigmentación, característica de este aceite de uña de gato hacen que el proceso de refinación sea más sencillo, ya que no fue necesaria la etapa de blanqueo, como se muestra en el diagrama 1. Aún así el contenido de carotenos del aceite refinado corresponde a $2.59 \mathrm{mg}$ por $\mathrm{kg}$ de aceite, que es más bajo que el reportado para el aceite de maíz, $5.4 \mathrm{mg} / \mathrm{kg}$ de aceite, (1).

En la Tabla II, se muestran algunas propiedades del aceite en las diferentes etapas de refinación. Donde se obtuvo una disminución de color y carotenos a través de los diferentes pasos. El índice de acidez, los ácidos grasos libres y el índice de peróxidos mostraron una disminución en
Tabla I

Características físicas y químicas del aceite crudo y refinado de Proboscidea parviflora

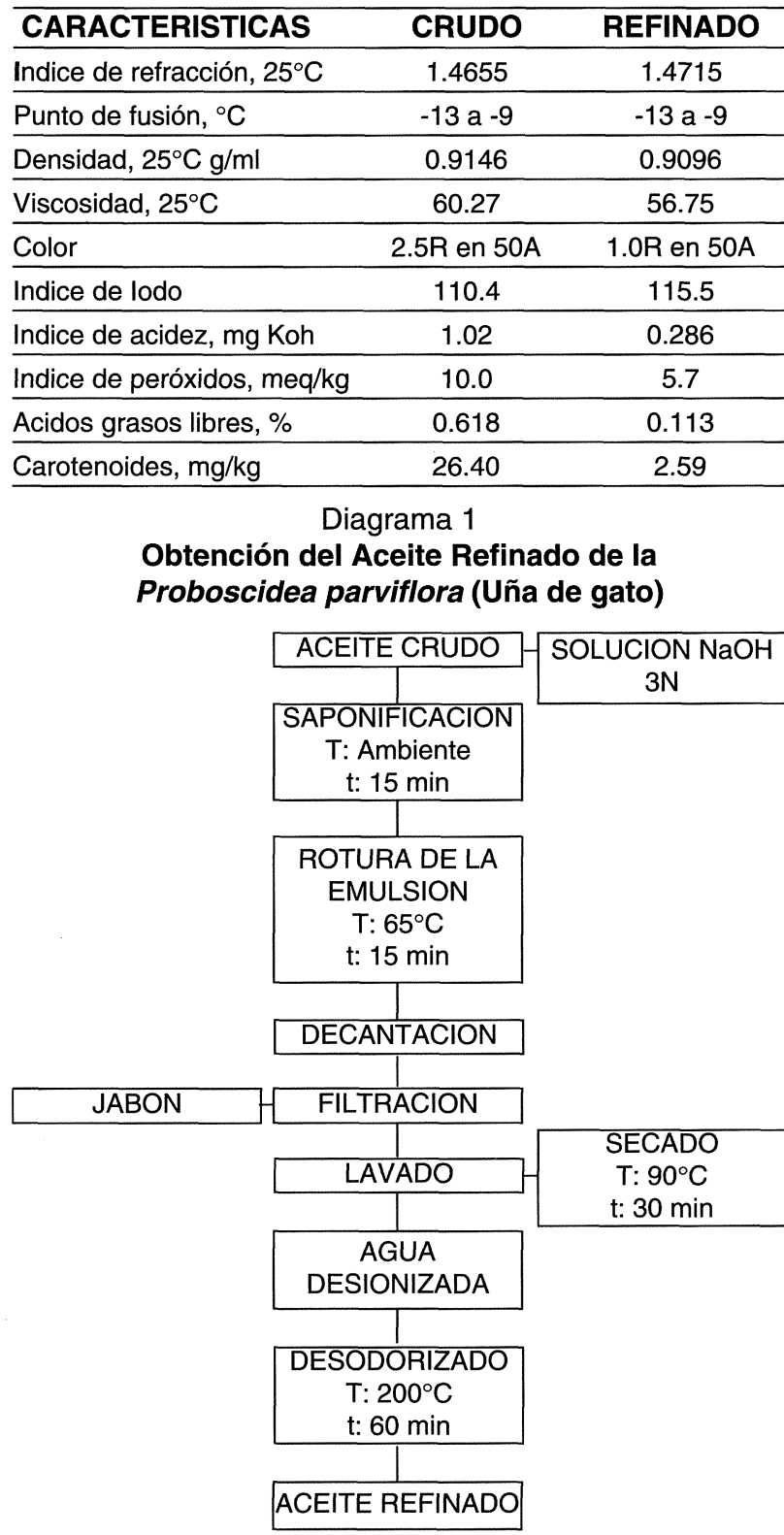

el proceso de neutralización y un ligero aumento en la etapa de desodorización provocado por la temperatura, aunque este aumento no fue significativo. Se observó también un incremento en el Indice de lodo y una disminución en la densidad del aceite. Se apreció que el índice de saponificación incrementó durante la refinación, esto es satisfactorio ya que tendió a aumentar en el proceso de desodorización. A pesar de estos incrementos, el aceite no manifestó oxidación y en este proceso sólo hubo pequeñas pérdidas de aceite, en porcentajes inferiores a la unidad. Se obtuvo un aceite libre de olores y sabores desagradables.

Al comparar el aceite de uña de gato con otros aceites de uso comercial como se muestra en la Tabla III se obser- 
Tabla II

Propiedades físicas y químicas de aceite de Proboscidea parviflora en las diferentes etapas de refinación

\begin{tabular}{|c|c|c|c|}
\hline \multirow[b]{2}{*}{ PROPIEDAD } & \multicolumn{3}{|c|}{$\begin{array}{llllll}A & C & E & \text { I } & \text { T } & E\end{array}$} \\
\hline & CRUDO & NEUTRALIZADO & DESODORIZADO \\
\hline Densidad $\mathrm{g} / \mathrm{ml}$ & 0.9146 & 0.9096 & 0.9096 \\
\hline Color R en 50A & 2.5 & 1.5 & 1.0 \\
\hline Indice de lodo & 110.4 & 114.3 & 115.5 \\
\hline Indice de acidez, mg Koh & 1.02 & 0.114 & 0.286 \\
\hline Indice de peróxidos, meq & 10.0 & 4.4 & 5.7 \\
\hline Acidos grasos libres, $\%$ & 0.525 & 0.060 & 0.113 \\
\hline Carotenoides, mg/kg & 27.40 & * & 2.59 \\
\hline Indice de saponificación & 157.9 & * & 179.2 \\
\hline
\end{tabular}

$\left.{ }^{\star}\right)$ No determinado

Tabla III

Características de aceites vegetales comparados con el de Proboscidea parviflora

\begin{tabular}{|c|c|c|c|c|}
\hline CARACTERISTICAS & SOYA $\left(^{*}\right)$ & AJONJOLI (*) & MAIZ $\left(^{*}\right)$ & P. Parviflora \\
\hline Acidos grasos libres, $\%$ & 0.5 & $\star \star$ & 1.5 & 0.61 \\
\hline Indice de lodo, mg/kg & 130 & 110.0 & 117.2 & 115.5 \\
\hline \multicolumn{5}{|l|}{ Indice de } \\
\hline saponificación, mg/kg & 192 & 191 & 190 & 179.2 \\
\hline Carotenoides, $\mathrm{mg} / \mathrm{kg}$ & 40.0 & ** & $\star \star$ & 27.4 \\
\hline Indice de refracción, $25^{\circ} \mathrm{C}$ & 1.474 & 1.473 & 1.472 & 1.4715 \\
\hline Densidad $25^{\circ} \mathrm{C}$ & 0.919 & 1.917 & 0.917 & 0.914 \\
\hline Viscosidad, $25^{\circ} \mathrm{C}$ & $\star \star$ & $\star \star$ & 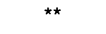 & 56.75 \\
\hline
\end{tabular}

${ }^{*}$ ) Bailey's Industrial and Fats Products (15) y este estudio.

$\left.{ }^{\star \star *}\right)$ No determinado

vó que el Indice de lodo es muy similar al de maíz, así los índices de refracción y la densidad son comparables también con estos aceites de uso ya tradicional. En las figuras 2 y 3 se muestran los cromatogramas obtenidos en la identificación de los ácidos grasos presentes en el aceite crudo y refinado de uña de gato respectivamente y las concentraciones se muestran en la Tabla IV. En ambos aceites hay un alto grado de insaturación $(86.29 \%)$ correspondiendo el $34.18 \%$ al ácido oléico y $52.11 \%$ al ácido linoléico, siendo éste el ácido graso predominante. Al comparar en la Tabla $V$ el perfil de ácidos grasos con otras especies oleaginosas se observa que el ácido linoléico se encuentra en una concentración similar en el aceite de girasol y en el de soya, mientras que el ácido oléico se encontró en concentración semejante al aceite de girasol. El palmítico está en concentración parecida al de maíz; la concentración del ácido linolénico en el aceite de uña de gato fue muy pequeña, esto hace que disminuya la probabilidad de que ocurra el fenómeno de reversión aumentando con esto la estabilidad y vida de almacenamiento del aceite. Además, se puede observar que la relación (oléico vs linoléico) de ácidos grasos insaturados tiene valores seme-
Tabla IV

Composición de ácidos grasos del aceite crudo y refinado de Proboscidea parviflora

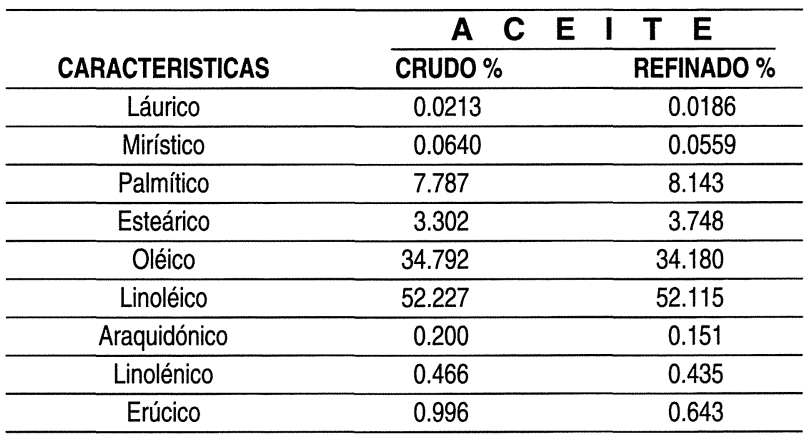

Tabla V

Comparación de la composición de algunos aceites comestibles comerciales con el de Proboscidea parviflora

\begin{tabular}{|c|c|c|c|c|}
\hline ACIDO GRASO & $\begin{array}{c}\text { GIRASOL }\left(^{*}\right) \\
\%\end{array}$ & $\begin{array}{c}\text { MAIZ (*) } \\
\%\end{array}$ & $\begin{array}{c}\text { SOYA }\left(^{*}\right) \\
\%\end{array}$ & $\begin{array}{c}\text { P. Parviflora } \\
\%\end{array}$ \\
\hline Palmítico & 3.6 & 7.8 & 11 & 8.14 \\
\hline Esteárico & 2.9 & 3.6 & 4.0 & 3.7 \\
\hline Oléico & 34.0 & 46.3 & 28.0 & 34.1 \\
\hline Linoléico & 57.1 & 41.7 & 50.0 & 52.1 \\
\hline Araquidónico & ** & 0.2 & $\star \star$ & 0.15 \\
\hline Linolénico & 0.2 & 0.8 & 8.0 & 0.43 \\
\hline
\end{tabular}

${ }^{*}$ ) Bailey's Industrial and Fats Products (15) y este estudio.

$\left({ }^{\star \star}\right)$ No determinado

jantes a el del aceite de girasol, y la de ácidos grasos saturados es similar a la del aceite de maíz. Basándose en estos resultados es evidente que una refinación sencilla bastó para que se obtuviera un aceite de buena calidad.

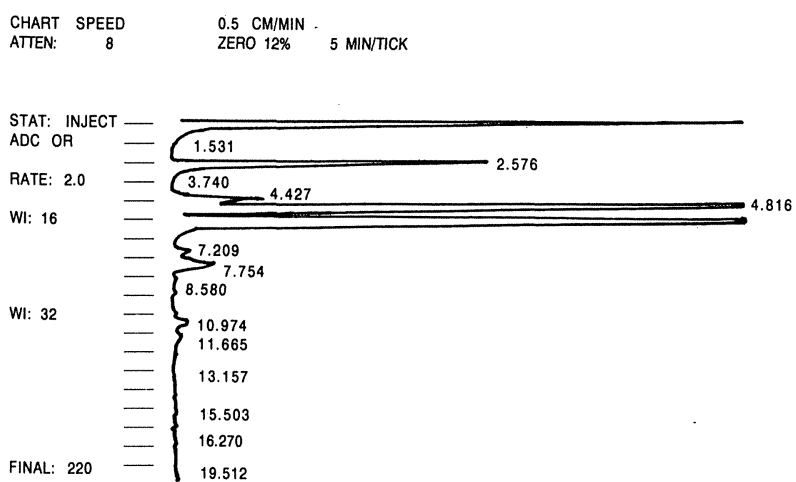

Figura 2

Cromatograma del aceite crudo de uña de gato (Proboscidea parviflora) 




Figura 3

Cromatograma del aceite refinado de uña de gato (Proboscidea parviflora)

\section{CONCLUSIONES}

Las semillas de uña de gato contienen mayor concentración de lípidos (39\%) que las de soya (18.7\%) y girasol (27.6\%). Al analizar sus características físicas y químicas se obtuvo un índice de iodo, relativamente alto reflejando un alto grado de insaturación que lo clasifica como un aceite semi-secante, localizado entre el aceite de ajonjolí y el aceite de maíz.

Otras características importantes del aceite es su índice de acidez que se encontró en cantidades pequeñas, y la baja pigmentación, que contribuyeron a que se lograra una refinación más sencilla. La cual se optimizó al utilizar hidróxido de sodio en una concentración $3 \mathrm{~N}$. Obteniéndose un aceite de buena calidad y estable. El aceite recuperado fue de color amarillo ámbar y sin olores desagradables, que al dejarlo a temperatura ambiente no manifestó rancidez $u$ oxidación. Otra propiedad interesante del aceite de uña de gato es que la prueba en frío es positiva, característica que debe tomarse en cuenta para la preparación de aceites para ensaladas o mayonesas.

\section{BIBLIOGRAFIA}

1. Arias, T. A., Gastelum, M. y Ruiz, A. (1980).- "Estudio físico y químico realizado al Aceite de Calabacilla, Cucurbita foetidissima". Tesis Universidad de Sonora.

2. Association of Official Analytical Chemist (1975).- 12 ava. Edition Washington, D. C

3. American Oil Chemist Society (1970).- "Tentative an Official Methods of Analysis".- Research Laboratory Swift and Co. Chicago, U.S.A

4. Berry, J. P. K., Breating, G. P., Nabhan, G. (1981).- "Proboscidea parviflora Domesticated a Potential Oilseed Crop for Arid Lands". J. Arid Environments 4, 147-160.

5. Benson, L. (1979).- "Plant Clasification".- D. C. Heath y Co. Toronto, Canadá.

6. Davidson, S. R., Passmore, J. F., Brock, Y., Truswell, A. S. (1975). "Human Nutrition and Dietetics".- Churchill Livingstone LTD., New York, Pags. 73-90, 142-150.

7. Chosh, A. and Earle, F. (1979).- "Seed lipid constituents of three species of Proboscidea".- Journal of Natural Products 42, 278-292.

8. Jones, Q. and Earle, F. (1966).- "Chemical Analysis of Seed II. Oil and Protein Content of 759 Species".- Economic Botanic 20, 127-155.

9. Nabham, G. P. (1981).- "Devel's Claw Domestication Evidence from Southwestern Indian Fields".- J. Etnobiol 1, 135-164.

10. Ortega, M. y Meza, J. (1987).- "Búsqueda e introducción de nuevos cultivos".- Informe Final, CICTUS, Universidad de Sonora.

11. Paur, F. (1952).- "Four Native New Mexico Plant Promișe as oil seed crops".- New Mexico Agricultural Experiment Station Press Bulletin U.S.A. 1064: 7 pp.

12. Sarh (1990).- "Cuaderno de Datos Básicos del Sector Agropecuario y Forestal".

13. Shreve, F. and Wiggins, I. (1964).- "Vegetation and Flora of the Sonoran Desert". Vol. I, pag. 191-243.

14. Wilet, T. J. (1988).- "Food Oil and Their Uses".- AVI Publishing Company, Inc., U.S.A.

15. Karl F., Frank A., Alexander J. and Swern D. Bayley's (1979).- "Industrial Oil and Fat Products".- Third edition.- Wiley of Sons, New York, Pag. 5872

(Recibido: Agosto 1992) 